\title{
Less may be more: shifting paradigm toward minimally invasive gastrectomy for locally advanced gastric cancer
}

\author{
Jashodeep Datta ${ }^{1}$, Vivian E. Strong ${ }^{2}$ \\ ${ }^{1}$ Division of Surgical Oncology, The Dewitt Daughtry Family Department of Surgery, University of Miami Miller School of Medicine, Sylvester \\ Comprehensive Cancer Center, Miami, FL, USA; ${ }^{2}$ Gastric and Mixed Tumor Service, Department of Surgery, Memorial Sloan Kettering Cancer \\ Center, New York, NY, USA \\ Correspondence to: Vivian E. Strong, MD. Gastric and Mixed Tumor Service, Department of Surgery, Memorial Sloan Kettering Cancer Center, 1275 \\ York Avenue, New York, NY 10065, USA. Email: strongv@mskcc.org. \\ Provenance: This is an invited article commissioned by the Academic Editor Dr. Zhiming Ma, MD (Division of Gastrointestinal Surgery, Department \\ of Surgery, The Second Hospital of Jilin University, Changchun, China). \\ Comment on: Yu J, Huang C, Sun Y, et al. Effect of Laparoscopic vs Open Distal Gastrectomy on 3-Year Disease-Free Survival in Patients With \\ Locally Advanced Gastric Cancer: The CLASS-01 Randomized Clinical Trial. JAMA 2019;321:1983-92.
}

Received: 10 September 2019; Accepted: 23 September 2019; Published: 26 November 2019.

doi: $10.21037 /$ tgh.2019.09.12

View this article at: http://dx.doi.org/10.21037/tgh.2019.09.12

Gastric cancer is the fifth most common malignancy and the third leading cause of cancer-related mortality worldwide (1). Despite evidence demonstrating improved outcomes with multimodality therapy (i.e., perioperative chemotherapy or adjuvant chemotherapy with/without radiotherapy), complete margin-negative surgical resection remains the mainstay of treatment (2). While open gastrectomy has remained the standard approach for gastric cancer resection globally, accumulating evidence over the last 2-3 decades from high-volume centers in Asia has indicated that minimally invasive gastrectomy (MIG) is an oncologically non-inferior approach in appropriately selected patients (3-8). The widespread adoption of MIG in Asia-particularly for early cancers located in the distal stomach-likely reflects a constellation of factors, such as higher incidence of gastric cancer, unique epidemiology (i.e., lower incidence of gastroesophageal junction tumors, lower rates of obesity-related cancers, higher $H$. pylori seroprevalence, etc.), and early detection screening programs (9). This paradigm shift also reflects a concerted effort by Asian surgeons to not only standardize the technical aspects of oncologic gastrectomy, but also translate these standards to the minimally invasive platform $(10,11)$. While adoption of MIG for gastric cancer has been more tentative in the United States (US) (12), recent data from higher volume US institutions have corroborated its safety and oncologic adequacy, particularly for early distal tumors (13).

In the East, a substantial body of level I evidence supports MIG for early gastric cancers (i.e., T1-2) located in the middle/lower third of the stomach, for which distal gastrectomy with limited lymphadenectomy is recommended by consensus Asian guidelines. For such early distal tumors, several randomized controlled trials (RCT) have demonstrated the typical benefits of the minimally invasive approach-namely decreased intraoperative blood loss, decreased pain scores, reduced length of stay, and improved quality of life $(3-5,6,11)$. Furthermore, the Korean KLASS-01 RCT demonstrated oncologic noninferiority (i.e., overall and cancer-specific survival) of MIG (n=705) compared with open gastrectomy $(n=711)$ for stage I gastric cancer. At a median follow-up of 100 months, 5 -year cancer-specific survival was $97.1 \%$ in the laparoscopic group and $97.2 \%$ in the open surgery group $(\mathrm{P}=0.9)$ (5). As such, MIG for early distal gastric cancer has become standard of care in Asia.

These impressive data in early gastric cancer set the stage for exploration of MIG for locally advanced disease, for which formal D2 lymphadenectomy is mandated in Asia. Prior to reporting of 3-year survival data from the Chinese Laparoscopic Gastrointestinal Surgery Study (CLASS)-01 trial (8), several retrospective case-control studies, meta- 
analyses, and four other Asian RCTs (14-17) had indicated that MIG was technically feasible in locally advanced gastric cancer. The initial iteration of the CLASS-01 trial, designed to evaluate non-inferiority of laparoscopic compared with open distal gastrectomy in locally advanced gastric cancer, reported on short-term surgical outcomes. The trial randomized 1,056 patients with clinical stage T2-4aN0-3M0 gastric cancer to either laparoscopic or open gastrectomy with D2 lymphadenectomy between September 2012 and December 2014 at 14 high-volume institutions in China. Postoperative morbidity and mortality were identical and compliance with D2 lymphadenectomy were exceptionally high in both groups. Moreover, MIG was associated with lower blood loss, decreased hospital stay, and earlier time to first flatus at the expense of longer operative times. Approximately $40 \%$ of patients in either arm received adjuvant 5-FU-based chemotherapy (reserved for stage II disease or higher by design) (3). In the discussion section of the manuscript, an onus was placed on the vast experience, expertise, and high-volume practice of the surgeons participating in this trial. Nonetheless, these data invoked a renewed appreciation for the feasibility of MIG for locally advanced gastric cancer.

The reporting of 3 -year survival data from this trial in $\mathcal{F} A M A$ earlier this year has catapulted this debate back into international dialog. At a median follow-up of nearly 38 months, the 3 -year disease-free survival (DFS) rates were $76.5 \%$ in the laparoscopic group and $77.8 \%$ in the open group, with an absolute difference of $-1.3 \%$ and a one-sided $97.5 \%$ CI that did not cross the prespecified non-inferiority threshold of $-10 \%$. These results were reproduced in their per-protocol analysis as well (i.e., after excluding patients who received total rather than distal gastrectomy, had inadequate D2 lymphadenectomy, or were switched to the other surgical approach preoperatively or intraoperatively). After adjusting for age, tumor size, pathologic $\mathrm{T}$ - and $\mathrm{N}$-classification, and adjuvant chemotherapy receipt, a mixed-effects Cox regression yielded a non-significant HR of 1.1 (95\% CI, 0.84-1.43) comparing laparoscopic with open distal gastrectomy. Moreover, the location and cumulative incidence of recurrence did not differ significantly between both arms. Three-year overall survival—a secondary outcome — was also similar between laparoscopic and open groups $(83.1 \%$ vs. $85.2 \%$, respectively). Although the absolute difference in gastric cancer-related death was $6.2 \%$ at 3 years favoring open gastrectomy, this did not reach statistical significance on post hoc exploratory analysis (8).
The CLASS consortium is to be congratulated on this impressive undertaking. These data represent the first level I evidence championing the oncologic non-inferiority of MIG in locally advanced gastric cancer. However, these data should be interpreted with two important caveats in mind: (I) a substantial proportion of patients were clinically overstaged; nearly a quarter of patients with clinical T2 tumors had pathologic T1 tumors, while nearly a third $(29.7 \%)$ of patients actually had pathologic stage I disease; (II) a post hoc sensitivity analysis revealed that, upon exclusion of patients with pathologic stage I tumors, noninferiority became nonsignificant, with the lower bound of the $97.5 \%$ CI $(-10.6 \%)$ crossing the pre-specified $10 \%$ noninferiority threshold. Clearly, the latter analysis is underpowered. Nonetheless, while acknowledging that clinical staging in Western/US centers is far from perfect, the applicability of these data to practice settings where endoscopic staging may be more robust is less certain.

Furthermore, the applicability of these data to Western and/or US patients remains unclear. First, management of locally advanced gastric cancer in the West/US is increasingly characterized by utilization of perioperative (even total neoadjuvant) chemotherapy, as evidenced in the FLOT4 (18) and MAGIC (19) trials. How translatable are these CLASS-01 data to the short-term (e.g., compliance with D2 lymphadenectomy and lymph node retrieval rates) and long-term (e.g., DFS and cancer-specific survival) oncologic outcomes in patients predominantly treated with neoadjuvant therapies? The German phase III Surgical Technique, Open versus Minimally-invasive gastrectomy After CHemotherapy (STOMACH) trial is currently underway, and will address this question (NCT02130726). Second, the incidence of proximal (i.e., cardia and gastroesophageal junction) gastric cancer is disproportionately on the rise in the West-particularly in North America. The CLASS-01 data cannot inform management for patients needing total gastrectomy. Third, the mean BMI in both arms in the CLASS-01 trial was 22.7 (8). With obesity rampant in the US, it is rare for even high-volume centers to see many patients with such BMIs. The impact of body habitus on the technical conduct of MIG cannot be overemphasized. These factors, in conjunction with the lower incidence of gastric cancer overall, the significant learning curve needed for technical proficiency in MIG (10), lack of centralization of gastric cancer to high-volume centers of excellence, and highly variable surgical and pathologic quality (12), may account for a rather tentative reception for these CLASS-01 data in 
the West/US until more globally applicable data become available.

\section{Acknowledgments}

None.

\section{Footnote}

Conflicts of Interest: The authors have no conflicts of interest to declare.

Ethical Statement: The authors are accountable for all aspects of the work in ensuring that questions related to the accuracy or integrity of any part of the work are appropriately investigated and resolved.

\section{References}

1. Bray F, Ferlay J, Soerjomataram I, et al. Global cancer statistics 2018: GLOBOCAN estimates of incidence and mortality worldwide for 36 cancers in 185 countries. CA Cancer J Clin 2018;68:394-424.

2. Roses RE, Datta J, You YN, et al. Multimodality treatment considerations in esophageal and gastric cancer: Are we making progress? Bull Am Coll Surg 2015;100:63-5.

3. Hu Y, Huang C, Sun Y, et al. Morbidity and Mortality of Laparoscopic Versus Open D2 Distal Gastrectomy for Advanced Gastric Cancer: A Randomized Controlled Trial. J Clin Oncol 2016;34:1350-7.

4. Katai H, Mizusawa J, Katayama H, et al. Short-term surgical outcomes from a phase III study of laparoscopyassisted versus open distal gastrectomy with nodal dissection for clinical stage IA/IB gastric cancer: Japan Clinical Oncology Group Study JCOG0912. Gastric Cancer 2017;20:699-708.

5. Kim HH, Han SU, Kim MC, et al. Effect of Laparoscopic Distal Gastrectomy vs Open Distal Gastrectomy on Long-term Survival Among Patients With Stage I Gastric Cancer: The KLASS-01 Randomized Clinical Trial. JAMA Oncol 2019;5:506-13.

6. Kim W, Kim HH, Han SU, et al. Decreased Morbidity of Laparoscopic Distal Gastrectomy Compared With Open Distal Gastrectomy for Stage I Gastric Cancer: Short-term Outcomes From a Multicenter Randomized Controlled Trial (KLASS-01). Ann Surg 2016;263:28-35.

7. Vinuela EF, Gonen M, Brennan MF, et al. Laparoscopic versus open distal gastrectomy for gastric cancer: a meta- analysis of randomized controlled trials and high-quality nonrandomized studies. Ann Surg 2012;255:446-56.

8. Yu J, Huang C, Sun Y, et al. Effect of Laparoscopic vs Open Distal Gastrectomy on 3-Year Disease-Free Survival in Patients With Locally Advanced Gastric Cancer: The CLASS-01 Randomized Clinical Trial. JAMA 2019;321:1983-92.

9. Russo A, Li P, Strong VE. Differences in the multimodal treatment of gastric cancer: East versus west. J Surg Oncol 2017;115:603-14.

10. Kang SY, Lee SY, Kim CY, et al. Comparison of Learning Curves and Clinical Outcomes between Laparoscopyassisted Distal Gastrectomy and Open Distal Gastrectomy. J Gastric Cancer 2010;10:247-53.

11. Kim MC, Jung GJ, Kim HH. Learning curve of laparoscopy-assisted distal gastrectomy with systemic lymphadenectomy for early gastric cancer. World J Gastroenterol 2005;11:7508-11.

12. Ecker BL, Datta J, McMillan MT, et al. Minimally invasive gastrectomy for gastric adenocarcinoma in the United States: Utilization and short-term oncologic outcomes. J Surg Oncol 2015;112:616-21.

13. Kelly KJ, Selby L, Chou JF, et al. Laparoscopic Versus Open Gastrectomy for Gastric Adenocarcinoma in the West: A Case-Control Study. Ann Surg Oncol 2015;22:3590-6.

14. Beyer K, Baukloh AK, Kamphues C, et al. Laparoscopic versus open gastrectomy for locally advanced gastric cancer: a systematic review and meta-analysis of randomized controlled studies. World J Surg Oncol 2019;17:68.

15. Cai J, Wei D, Gao CF, et al. A prospective randomized study comparing open versus laparoscopy-assisted D2 radical gastrectomy in advanced gastric cancer. Dig Surg 2011;28:331-7.

16. Park YK, Yoon HM, Kim YW, et al. Laparoscopy-assisted versus Open D2 Distal Gastrectomy for Advanced Gastric Cancer: Results From a Randomized Phase II Multicenter Clinical Trial (COACT 1001). Ann Surg 2018;267:638-45.

17. Shi $Y, X u X, Z$ hao $Y$, et al. Short-term surgical outcomes of a randomized controlled trial comparing laparoscopic versus open gastrectomy with D2 lymph node dissection for advanced gastric cancer. Surg Endosc 2018;32:2427-33.

18. Al-Batran SE, Homann N, Pauligk C, et al. Perioperative chemotherapy with fluorouracil plus leucovorin, oxaliplatin, and docetaxel versus fluorouracil or capecitabine plus cisplatin and epirubicin for locally advanced, resectable gastric or gastro-oesophageal junction 
adenocarcinoma (FLOT4): a randomised, phase 2/3 trial. Lancet 2019;393:1948-57.

19. Cunningham D, Allum WH, Stenning SP, et al.

doi: $10.21037 / \operatorname{tgh} .2019 .09 .12$

Cite this article as: Datta J, Strong VE. Less may be more: shifting paradigm toward minimally invasive gastrectomy for locally advanced gastric cancer. Transl Gastroenterol Hepatol 2019;4:79.
Perioperative chemotherapy versus surgery alone for resectable gastroesophageal cancer. $\mathrm{N}$ Engl J Med 2006;355:11-20. 